\title{
A discrete phase problem in reconstruction of signals in space-rocket hardware
}

\author{
A.A. Kuleshova ${ }^{1}$, E.A. Shchelokov ${ }^{1}$ \\ ${ }^{1}$ Samara National Research University, 34 Moskovskoe Shosse, 443086, Samara, Russia
}

\begin{abstract}
Reconstruction of information hidden in vector signal phases does not lose its relevance. Sets of vectors $\Phi=\left\{\varphi_{i}\right\}_{i=1}^{n}$, called frames, in a space $C^{\mathrm{m}}\left(R^{\mathrm{m}}\right)$ can be used for theoretical research of phase retrieval. The article shows that phase retrieval is equivalent to phaseless reconstruction. Examples are considered in $R^{\mathrm{m}}$ and $C^{\mathrm{m}}$, for which sets of vectors $\Phi=\left\{\varphi_{i}\right\}_{i=1}^{n}$ that simultaneously carry out phase retrieval and phaseless reconstruction are constructed.
\end{abstract}

Keywords: phase retrieval; phaseless reconstruction; frame; complement property; weak phase retrieval; generic frame

\section{Introduction}

A search of the fast algorithms for phaseless signal reconstruction is topical now. The main property of frames, which makes them so useful in applied tasks, is their redundancy. A well-chosen frame can provide numerical stability for signal recovery and obtaining important characteristics of the signal [1]. A family of frames recovers the signal by absolute values of frame coefficients in polynomial time.

It is shown that in the real case under certain conditions a generic frame consisting of (2m-1)-vectors can recover the signal without phases. The similar result was obtained in the complex space for $(4 \mathrm{~m}-2)$-vectors.

Along with the "phaseless reconstruction", another version of the discrete phase problem statement - "phase retrieval" - is considered. The issue of their equivalence is raised and partially resolved.

The present work continues this line of research and gives examples of signal recovery in small-dimension spaces.

\section{A Discrete Phase Problem in Reconstruction of Signals in Space-Rocket Hardware}

Now the problem of reduction of the mass of cable systems in spacecraft is widely known. In this connection we offer for consideration an option of replacement of the cable system by a radio channel [2].

Widespread introduction of wireless devices has become possible as a result of improvement and reduction in cost of electronic components. Modern chips, which are used in construction of wireless networks, only require connection of several passive components and program setup.

In connection with the above-mentioned it is reasonable to consider the introduction of wireless technologies into spacerocket hardware as one of ways to reduce the mass and complexity of the cable system.

Let's consider, as an example, a signal transmitted by a radio channel with a modulation of an OFDM type. The main advantage of the chosen method is that the signal propagation delay is much less, than time of transfer of a symbol in auxiliary carriers as compared to other types of modulation. That allows implementing more stable transfer of information under conditions of symbols overlapping in the course of rereflections of the signal.

Figure 1 shows a distribution model of levels of signals from a different number of access points for a case when a set of blocks is arranged inside a spacecraft. The model is presented in a two-dimensional form, however, as is obvious from distribution of levels of signals, 4 access points is enough to provide communication of all blocks among themselves, including by relaying.

As is evident from figure 1, the signal levels at the border of the external and internal parts of the compartment do not exceed minus $30 \mathrm{dBm}$ (blue color:-30 to $-40 \mathrm{dBm}$, green color:-40 to $-50 \mathrm{dBm}$ ) relative to $0 \mathrm{dBm}$ at the antenna exit. If we take into account that aluminum has a shielding factor of $70 \mathrm{dBm}$ (for the thickness of $5 \mathrm{~mm}$ ) we obtain the coefficient of signal attenuation outside the working zone equal to $100 \mathrm{dBm}$. If an additional protection is necessary, it is enough not to allow blocking direct visibility of the transmitting part and the compartment border, which will increase attenuation by $30-40 \mathrm{~dB}$ [2].

In OFDM modulation, data are distributed among a great number of auxiliary carriers, that's why it is necessary to recover the information lost in several subchannels for further data handling. A Search of the signal recovery algorithms is topical now. Sampling and quantization of the analog signal lead to consideration of the signal as an element of a finite-dimensional space $V$. By the orthonormalized basis (ONB) $\left\{u_{i}\right\}_{i=1}^{m}$, the "signal" $v \in \mathrm{V}$ can be uniquely represented in the form of the sum: $v=\sum_{i=1}^{m}\left\langle v, u_{i}\right\rangle u_{i}$. Actual measurements prove real, and the gap between $\left\langle v, u_{i}\right\rangle$ and amplitudes of measurements $\left|\left\langle v, u_{i}\right\rangle\right|$ proves insuperable in signal reconstruction [1, P. 280], [4, P.281]. 


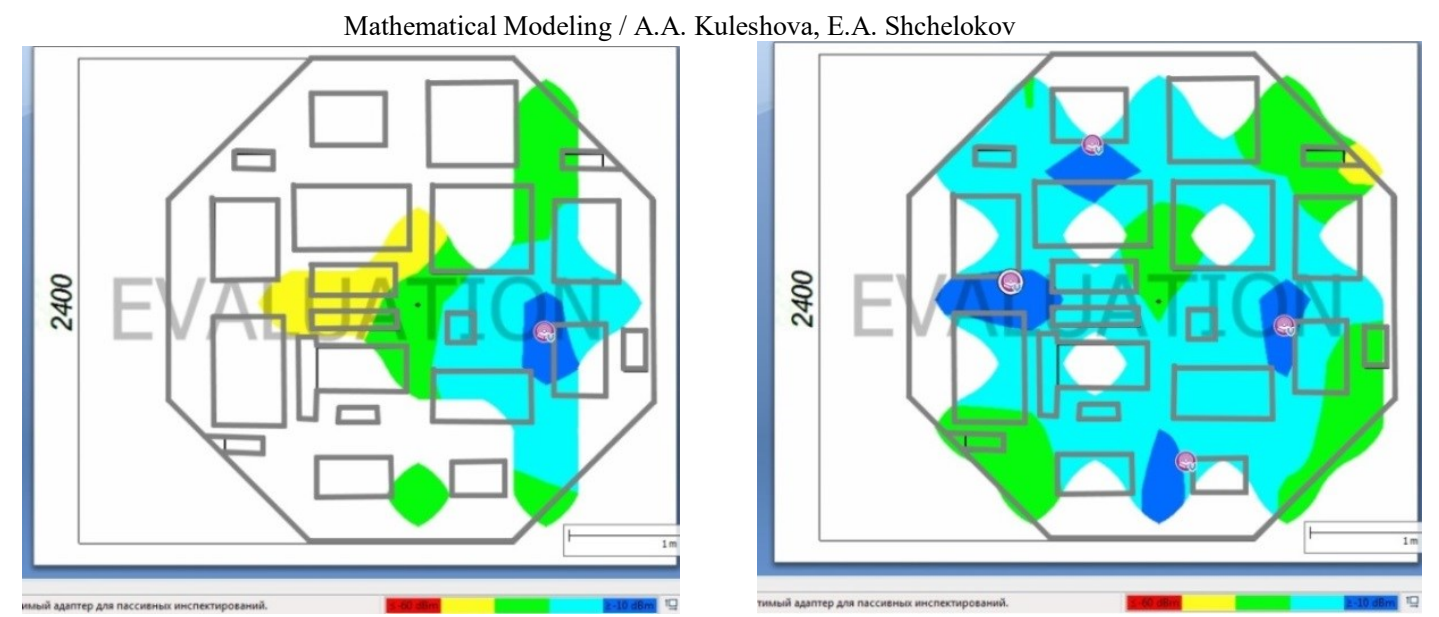

Fig. 1. Distribution of the signal from one access point (on the left) and from four access points (on the right).

In recent years, significant amount of works has been devoted to solution of the following task: to construct such systems of "measuring" vectors $\Phi=\left\{\varphi_{i}\right\}_{i=1}^{n}$ that allow recovering a signal $v \in \mathrm{V}$ by a set of real numbers $\left|\left\langle, \varphi_{i}\right\rangle\right|$.

Such task has no decision in the ONB class.

The main problem set in [3] is still far from final solution. It is to find necessary and sufficient conditions for a system of representation vectors $\Phi=\left\{\varphi_{i}\right\}_{i=1}^{n}$ (so-called "measuring vectors"), which provide injectivity and stability of mapping of "amplitude measurement" of the signal $\mathrm{x}$

$$
(A(x))(i):=\left.\left\langle x, \varphi_{i}\right\rangle\right|^{2}
$$

We have proved that exact recovery of the signal (to the unimodular multiplier) is theoretically possible if complete redundant systems are used as a representation system [2, P. 354]. Frames are such redundant systems.

In 2006, Balan/Casazza/Edidin [4,5] defined one of versions of the discrete phase problem, which they called "phaseless reconstruction". It was shown that in the real case a generic frame consisting of (2m-1)-vectors can do phaseless reconstruction under certain conditions. The similar result was obtained for (4m-2)-vectors in the complex space.

\section{Frames}

Let $H^{\mathrm{m}}$ be a space $R^{\mathrm{m}}$ or $C^{\mathrm{m}}$.

Definition 1. A family of vectors $\Phi=\left\{\varphi_{i}\right\}_{i=1}^{n}$ is called a frame of a Hilbert space $H^{\mathrm{m}}$ if there are such constants $0<\mathrm{A} \leq \mathrm{B}<$ $\infty$ that for all $x \in H^{m}$ the following inequalities are achieved:

$$
A\|x\|^{2} \leq \sum_{i=1}^{n}\left|\left\langle x, f_{i}\right\rangle\right|^{2} \leq B\|x\|^{2} .
$$

A and B are called frame bounds. The greatest of the lower bounds is called the optimum lower bound, and the smallest of the upper bounds is the optimum upper bound. If $\mathrm{A}=\mathrm{B}$, than the frame is called $\mathrm{A}$-tight and if $\mathrm{A}=\mathrm{B}=1$, it is called a Parseval-Steklov frame.

The numbers $\left\{\left\langle x, \varphi_{i}\right\rangle\right\}_{i=1}^{n}$ are called frame coefficients.

If all frame elements have the same norm than such frames are called uniform ones.

In the finite-dimensional space the notion of a frame is equivalent to the notion of completeness of a system, that is to the equality $\operatorname{span}\left\{\varphi_{i}\right\}_{i=1}^{n}=H^{m}[5]$.

Definition 2. Let $\Phi=\left\{\varphi_{i}\right\}_{i=1}^{n}$ be a frame. The linear mapping:

$$
T: H^{m} \rightarrow H^{n}, \quad T(x)=\left\{\left\langle x, \varphi_{i}\right\rangle\right\}_{i=1}^{n}
$$

is called an analysis operator.

Definition 3. The linear mapping:

$$
T^{*}: H^{n} \rightarrow H^{m}, T^{*}\left(\left\{c_{i}\right\}_{i=1}^{n}\right)=\sum_{i=1}^{n} c_{i} \varphi_{i}
$$

is called a synthesis operator.

The composition of $T$ and $T^{*}$ defines a frame operator, which is a positive, self-conjugate reversible operator:

$$
S=T^{*} T: H^{m} \rightarrow H^{m}: S x=T^{*} T x=\sum_{i=1}^{n}\left\langle x, \varphi_{i}\right\rangle \varphi_{i} .
$$

It provides the exact formula for reconstruction:

$$
x=\sum_{i=1}^{n}\left\langle x, \varphi_{i}\right\rangle S^{-1} \varphi_{i} .
$$

Definition 4. A family of vectors $\Phi=\left\{\varphi_{i}\right\}_{i=1}^{n}$ is a uniform equiangular tight frame if 
1) $\exists \beta>0:\left\|\varphi_{i}\right\|=\beta \quad \forall i=\overline{1, n}$;

2) $\exists c>0$ : for any pair of frame vectors $\varphi_{j}$ and $\varphi_{k}, \mathrm{j} \neq \mathrm{k}$, we have:

$$
\left\langle\varphi_{j}, \varphi_{k} \mid\right\rangle=c \text {. }
$$

It is known that there is an upper bound for a number of vectors in the uniform equiangular tight frame $\Phi=\left\{\varphi_{i}\right\}_{i=1}^{n}$ on the mdimensional Hilbert space H. In the real case it is $n \leq \frac{m(m+1)}{2}$, in the complex case it is $n \leq m^{2}$ ([6], [7]). Creation of the maximum number of vectors for the uniform equiangular tight frame is a very complex and unresolved problem in the theory of frames.

Let us consider a non-linear mapping $P$, which transfers the vector into a set of the absolute values of frame coefficients:

$$
P: H \rightarrow l^{2}(I), P(x)=\left\{\left|\left\langle x, \varphi_{i}\right\rangle\right|\right\}_{i=1}^{n}
$$

Definition 5. The frame $\left\{\varphi_{i}\right\}_{i=1}^{n}$ is called generic if $\left\{\varphi_{i}\right\}_{i=1}^{n} \subset L \in U$, where $\mathrm{U}$ is the Zariski open set and $U \subset G r(m, n)$.

Theorem 1 [8,9]. Let $\Phi=\left\{\varphi_{i}\right\}_{i=1}^{n} \subseteq C^{m}$ and the mapping $A: C_{r}^{m}=C^{m} / T^{1} \rightarrow R^{m}$ be defined by $(A(x))(i):=\left|\left\langle x, \varphi_{i}\right\rangle\right|^{2}$, $i=1, . ., n$.

Let us consider $\left\{\varphi_{i} \varphi_{i}^{*} u\right\}_{i=1}^{n}$ as vectors of the space $R^{2 m}$. Let $S(u):=\operatorname{span}_{R}\left\{\varphi_{i} \varphi_{i}^{*} u\right\}_{i=1}^{n}$. The following statements are equivalent:

(a) $A$ is injective.

(b) $\operatorname{dim} S(u) \geq 2 n-1$ for every $u \in C^{m} \backslash\{0\}$.

(c) $S(u)=\operatorname{span}_{R}\{i u\}^{\perp}$ for every $u \in C^{m} \backslash\{0\}$.

Definition 6. The family of vectors $\Phi=\left\{\varphi_{i}\right\}_{i=1}^{n}$ in $\mathrm{H}^{\mathrm{m}}$ has the complement property if for any $I \subseteq\{1, \ldots, \mathrm{n}\}$, either $\left\{\varphi_{i}\right\}_{i \in I}$, or $\left\{\varphi_{i}\right\}_{i \in I^{C}}$ is complete in $\mathrm{H}^{\mathrm{m}}[10]$.

Definition 7. The family of vectors $\left\{\varphi_{i}\right\}_{i=1}^{n} \subseteq R^{m}$ is called a set with a full spark, if every its subset of $m$ vectors is complete in $R^{m}$ [10].

Lemma 1. Every set with the full spark $\Phi=\left\{\varphi_{i}\right\}_{i=1}^{n}$ in $R^{m}$ with $n \geq 2 m-1$ satisfies the complement property.

Proof. Let's assume the contrary: there is such $S \subseteq\{1, . ., n\}$ that neither $\left\{\varphi_{i}\right\}_{i \in S}$ nor $\left\{\varphi_{i}\right\}_{i \in S^{C}}$ are not complete in $R^{m}$.

By definition of the full spark, from this it follows that $|S|<m-1$ and $\mid S^{C}<m-1$, that is $n<2 m-2$, which contradicts the condition.

Theorem 2. In the real case if $\Phi=\left\{\varphi_{i}\right\}_{i=1}^{n}$ in $R^{m}$ and $n \leq 2 m-2$, then mapping $A$ is not injective.

If $n=2 m-1$, then mapping $A$ is injective if and only if when $\Phi=\left\{\varphi_{i}\right\}_{i=1}^{n}$ is a full spark.

Proof. If $n \leq 2 m-2$, then the set $\{1, . ., n\}$ can be divided into sets $S$ and $S^{C}$ such that the cardinality of each would not exceed $m-1$. None of the sets $\left\{\varphi_{i}\right\}_{i \in S},\left\{\varphi_{i}\right\}_{i \in S^{C}}$ can be complete.

If $n=2 m-1$ and $\Phi=\left\{\varphi_{i}\right\}_{i=1}^{n}$ is a full spark, then the injectivity of $A$ follows from lemma 1 and theorem 1.

And vice-versa, if $A$ is injective, then $\Phi=\left\{\varphi_{i}\right\}_{i=1}^{n}$ is an alternatively full family. Let's take an arbitrary subset $S \subseteq\{1, . ., n\}$ with $|S|=m$. Then $\left|S^{C}\right|=m-1$ and $\left\{\varphi_{i}\right\}_{i \in S^{C}}$ can't be full. Therefore, $\left\{\varphi_{i}\right\}_{i \in S}$ is full, and $\Phi=\left\{\varphi_{i}\right\}_{i=1}^{n}$ is a full spark.

The exact minimum bound is unknown for the complex case. Besides, in the real case there is a simple direct method for checking infectivity of the mapping $A$ for the corresponding frame [7].

Theorem $3[4,11]$.

(a) If $H \in R^{m}, n \geq \frac{m(m+1)}{2}$ and $\Phi=\left\{\varphi_{i}\right\}_{i=1}^{n}$ is a generic frame, the nonlinear map $P$ is injective. Then the vector $\mathrm{x} \in \mathrm{H}$ can be reconstructed (up to a sign) from the set $\left\{\left|\left\langle x, \varphi_{i}\right\rangle\right|\right\}_{i=1}^{n}$ of absolute values of the frame coefficients in a polynomial number $\left(\mathrm{O}\left(\mathrm{m}^{6}\right)\right)$ of steps.

(b) If $H \in C^{m}, n \geq m^{2}$ and $\Phi=\left\{\varphi_{i}\right\}_{i=1}^{n}$ is a generic frame, the nonlinear map $P$ is injective. Then the vector $\mathrm{x} \in \mathrm{H}$ can be reconstructed (up to multiplication by a root of unity) from the set $\left\{\left|\left\langle x, \varphi_{i}\right\rangle\right|\right\}_{i=1}^{n}$ of absolute values of the frame coefficients in a polynomial number $\left(\mathrm{O}\left(\mathrm{m}^{6}\right)\right)$ of steps.

\section{About Equivalence of Phase Retrieval and Phaseless Reconstruction}

Let $x=\left(a_{1}, a_{2}, \ldots, a_{m}\right)$ and $y=\left(b_{1}, b_{2}, \ldots, b_{m}\right)$ be vectors in a space $H^{\mathrm{m}}$.

Definition 1: For the phase of number $z \in C^{\mathrm{m}}$, we take the value of the angle $\varphi=p h z_{i}+2 \pi k, k \in Z$, defining the deviation of the radius vector of the point on the plane, corresponding to $z \in C^{\mathrm{m}}$, from the real axis in $C^{\mathrm{m}}$. In the real case, the phase in $R^{\mathrm{m}}$ is equal to 0 or $\pi$.

We shall say that $x, y$ have the same phases if: 


$$
\text { ph } a_{i}=\text { ph } b_{i}, i=1,2, \ldots, m \text {. }
$$

Definition 2. Let $\Phi=\left\{\varphi_{i}\right\}_{i=1}^{n}$ be a family of vectors in $H^{\mathrm{m}}$ (respectively, $\left\{P_{i}\right\}_{i=1}^{n}$ be a family of projections on $H^{\mathrm{m}}$ ) satisfying the following property: for every $x, y$ the following condition is satisfied:

(Respectively,

$$
\left|\left\langle x, \varphi_{i}\right\rangle\right|=\left\langle y, \varphi_{i}\right\rangle, \text { for all } i=1,2, \ldots, n \text {. }
$$

$$
\left.\left\|P_{i} x\right\|=\left\|P_{i} y\right\|, \text { for all } i=1,2, \ldots, n\right) \text {. }
$$

Then

1) If there is a $|\theta|=1$ such that $x$ and $\theta y$ have the same phases, one can say $\Phi=\left\{\varphi_{i}\right\}_{i=1}^{n}$ does phase retrieval (respectively, $\left\{P_{i}\right\}_{i=1}^{n}$ does phase retrieval).

2) If there is a $|\theta|=1$ such that $x=\theta y$, one can say $\Phi=\left\{\varphi_{i}\right\}_{i=1}^{n}$ does phaseless reconstruction. (Respectively, $\left\{P_{i}\right\}_{i=1}^{n}$ does phaseless reconstruction.)

Definition 3. Let us call a family of vectors $\Phi=\left\{\varphi_{i}\right\}_{i=1}^{n}$ in $\mathrm{H}^{\mathrm{m}}$ an alternatively full one, if for any $I \subseteq\{1, \ldots, \mathrm{n}\}$, either $\left\{\varphi_{i}\right\}_{i \in I}$, or $\left\{\varphi_{i}\right\}_{i \in I}$ is complete in $\mathrm{H}^{\mathrm{m}}$.

If $\left\{\varphi_{i}\right\}_{i=1}^{n}$ retrieves phases in $\mathrm{H}^{\mathrm{m}}$ then $\operatorname{span}\left\{\varphi_{i}\right\}_{i=1}^{n}=\mathrm{H}^{\mathrm{m}}$. This means that $\left\{\varphi_{i}\right\}_{i=1}^{n}$ is a frame in the space $\mathrm{H}^{\mathrm{m}}$. Otherwise, there exists $0 \neq x \in \mathrm{H}^{\mathrm{m}}$ such that $\left\langle x, \varphi_{i}\right\rangle=\left\langle y, \varphi_{i}\right\rangle=0, i=1,2, \ldots, n$, while phases of vectors $x$ and 0 are not the same.

If $x=\left(a_{1}, a_{2}, \ldots, a_{m}\right)$ and $y=\left(b_{1}, b_{2}, \ldots, b_{m}\right)$ have the same phases then $a_{i}=0$ if and only if $b_{i}=0$, for the phase of 0 is not determined.

Theorem 1. Let $\Phi=\left\{\varphi_{i}\right\}_{i=1}^{n}$ be a set of vectors in $R^{\mathrm{m}}$. The mapping $A: R^{m} /\{ \pm 1\} \rightarrow R^{n} \quad(n>m)$ is defined by $(A(x))(i):=\left|\left\langle x, \varphi_{i}\right\rangle\right|^{2}, i=1, . ., n$. If $\Phi=\left\{\varphi_{i}\right\}_{i=1}^{n}$ does phaseless reconstruction, then it has a complement property. In the real case these concepts are equivalent.

Proof. $(\Rightarrow)$ Assume that $\Phi$ fails the complement property. Then there exists $I \subseteq\{1, . ., n\}$ such that neither $\left\{\varphi_{i}\right\}_{i \in I}$, nor $\left\{\varphi_{i}\right\}_{i \in I^{C}}$ is complete in $R^{m}$.

We choose nonzero vectors $u, v \in R^{m}$ such that $\left\langle u, \varphi_{i}\right\rangle=0$ for all $i \in I$ and $\left\langle v, \varphi_{i}\right\rangle=0$ for all $i \in I^{C}$. For every $i$ we then have:

$$
\left|\left\langle u \pm v, \varphi_{i}\right\rangle\right|^{2}=\left|\left\langle u, \varphi_{i}\right\rangle\right|^{2} \pm 2\left\langle u, \varphi_{i}\right\rangle \overline{\left\langle v, \varphi_{i}\right\rangle}+\left|\left\langle v, \varphi_{i}\right\rangle\right|^{2}=\left|\left\langle u, \varphi_{i}\right\rangle\right|^{2}+\left|\left\langle v, \varphi_{i}\right\rangle\right|^{2} .
$$

From this it follows that $\left.\left|u+v, \varphi_{i}\right\rangle\right|^{2}=\left|\left\langle u-v, \varphi_{i}\right\rangle\right|^{2}$ for every $i$, and $A(u+v)=A(u-v)$. Moreover, since $u$ and $v$ are nonzero by assumption, then $u+v \neq \pm(u-v)$. Thus there is no phaseless reconstruction.

$(\Leftarrow)$ Assume that $\Phi=\left\{\varphi_{i}\right\}_{i=1}^{n}$ fails phaseless reconstruction. That means there exist vectors $x, y \in R^{m}$ such that $x \neq \pm y$ and $A(x)=A(y)$. Take $I:=\left\{i:\left\langle x, \varphi_{i}\right\rangle=-\left\langle y, \varphi_{i}\right\rangle\right\}$.

We have: $\left\langle x+y, \varphi_{i}\right\rangle=0$ for every $i \in I$. Otherwise if $i \in I^{C}$, we have $\left\langle x, \varphi_{i}\right\rangle=\left\langle y, \varphi_{i}\right\rangle$ and then $\left\langle x-y, \varphi_{i}\right\rangle=0$. According to the assumption, $x \neq \pm y$, therefore $x+y \neq 0$ and $x-y \neq 0$. Thus, neither $\left\{\varphi_{i}\right\}_{i \in I}$ or $\left\{\varphi_{i}\right\}_{i \in I^{c}}$ are complete in $R^{m}$.

In $R^{\mathrm{m}}$ the phase of a vector can be equal to 0 or $\pi$. Coordinates of the vectors have the same phases if signs of the coordinates of the vector $x$ are the same as those of the vector $y$. At the same time the phase of 0 is not defined. That is, if $x=\left(a_{1}, a_{2}, \ldots, a_{m}\right)$ and $y=\left(b_{1}, b_{2}, \ldots, b_{m}\right)$, then $x, y$ have the same phase in the following cases:

1) If $a_{i} \neq 0 \neq b_{i}$, then $a_{i} b_{i}>0$.

2) If $a_{i}=0$, then corresponding to it $b_{i}=0$ (it is symmetric: if $b_{i}=0$, then corresponding to it $a_{i}=0$ ).

Otherwise, the vectors have different phases.

Then, if we are given two vectors, so as to define whether their phases are equal or not it is necessary:

1) To check equality of all indices of zero coordinates of the vectors. If all indexes of zero coordinates of the first vector correspond to the indexes of the second vector (and vice versa), then it is necessary to check 2), otherwise, the vectors have different phases.

2) For nonzero coordinates to check fulfillment of the following condition: if $a_{i} b_{i}>0 \Rightarrow$ the vectors have the same phases, and if $a_{i} b_{i}<0$ then the vectors have different phases.

Definition 1 in the real case will mean:

Let $\Phi=\left\{\varphi_{i}\right\}_{i=1}^{n}$ be a set of vectors in $R^{\mathrm{m}}$, satisfying the following property: for every $x, y$ the following condition is fulfilled:

$$
\left|\left\langle x, \varphi_{i}\right\rangle\right|=\mid\left\langle y, \varphi_{i}\right\rangle, i=1,2, \ldots, n .
$$


Then,

1) $\Phi=\left\{\varphi_{i}\right\}_{i=1}^{n}$ does phases reconstruction if there exist $\theta= \pm 1$ such that

a) For $\theta=1$ the vectors $\mathrm{x}$ and $y$ have the same phase.

b) For $\theta=-1$ the vectors $\mathrm{x}$ and $-y$ have the same phase.

2) $\Phi=\left\{\varphi_{i}\right\}_{i=1}^{n}$ does phaseless reconstruction if there exists $\theta= \pm 1$ such that

c) For $\theta=1 \Rightarrow x=y$.

d) For $\theta=-1 \Rightarrow x=-y$.

Theorem 2. Let $\Phi=\left\{\varphi_{i}\right\}_{i=1}^{n}$ be a set of vectors in $R^{\mathrm{m}}$. The mapping $A: R^{m} /\{ \pm 1\} \rightarrow R^{n}(n>m)$ is defined by equations $(A(x))(i):=\left.\left\langle x, \varphi_{i}\right\rangle\right|^{2}, i=1, . ., n$. If $\Phi=\left\{\varphi_{i}\right\}_{i=1}^{n}$ does phase retrieval, then it has the complement property. In the real case these concepts are equivalent.

Proof. Assume that $\Phi$ does phase retrieval, but fails phaseless reconstruction. Assume that the set $\Phi=\left\{\varphi_{i}\right\}_{i=1}^{n}$ fails complement property, that is there exists $I \subseteq\{1, . ., n\}$ such that neither $\left\{\varphi_{i}\right\}_{i \in I}$, nor $\left\{\varphi_{i}\right\}_{i \in I^{C}}$ is complete in $R^{m}$.

Let us choose nonzero vectors $x=\left(a_{1}, a_{2}, \ldots, a_{m}\right), y=\left(b_{1}, b_{2}, \ldots, b_{m}\right) \in R^{m}$ such that $\left\langle x, \varphi_{i}\right\rangle=0$ for all $i \in I$ and $\left\langle y, \varphi_{i}\right\rangle=0$ for all $i \in I^{C}$. Then for some $i$ either $\left\langle x, \varphi_{i}\right\rangle=0$, or $\left\langle y, \varphi_{i}\right\rangle=0$. Fix $c \neq 0$, so that for every $1 \leq i \leq n$

$$
\left|\left\langle x+c y, \varphi_{i}\right\rangle\right|=\left|\left\langle x-c y, \varphi_{i}\right\rangle\right|
$$

Then

$$
\left|\left\langle x+c y, \varphi_{i}\right\rangle\right|^{2}=\left|\left\langle x-c y, \varphi_{i}\right\rangle\right|^{2}
$$

By assumption, $\Phi$ does phase retrieval, and it means that there exists $|\theta|=1$ such that $(x+c y)$ and $\theta(x-c y)$ have the same phases. Let's assume that there exists $1 \leq i_{0} \leq m$ such that $a_{i 0} \neq 0 \neq b_{i 0}$ and let $c=\frac{-a_{i 0}}{b_{i 0}}$. Then

and

$$
(x+c y)_{i 0}=a_{i 0}+c b_{i 0}=a_{i 0}+\frac{-a_{i 0}}{b_{i 0}} b_{i 0}=0
$$

$$
(x-c y)_{i 0}=a_{i 0}-c b_{i 0}=a_{i 0}-\frac{-a_{i 0}}{b_{i 0}} b_{i 0}=2 a_{i 0} \neq 0 .
$$

But it is impossible because if $x$ and $y$ have the same phases, then $a_{i}=0$ if and only if $b_{i}=0$.

As the vectors $x$ and $y$ are nonzero, then the last two equalities are possible if and only if either $a_{i}=0$, or $b_{i}=0$, $1 \leq i \leq m$. Let $I=\left\{1 \leq i \leq m: b_{i}=0\right\}$ and $\left\{e_{i}\right\}_{i=1}^{m}$ be an orthonormalized basis in $R^{m}$. Then

$$
x+y=\sum_{i \in I} a_{i} e_{i}+\sum_{i \in I^{C}} b_{i} e_{i} \text { and } x-y=\sum_{i \in I} a_{i} e_{i}+\sum_{i \in I^{C}}\left(-b_{i}\right) e_{i}
$$

Then there exists $|\theta|=1$ such that $(x+y)$ and $\theta(x-y)$ have the same phases and they are equal. We have arrived at a contradiction.

Let's consider an example in $R^{2}$. Let $x=\left(a_{1}, a_{2}\right) \neq(0,0)$ and $y=\left(b_{1}, b_{2}\right) \neq(0,0)$.

For $\Phi=\left\{\varphi_{i}\right\}_{i=1}^{3}$, we take the Mercedes-Benz frame in $R^{2}$, consisting of 3 unit vectors located at an angle of $120^{\circ}$ (Fig. 2 ): $\varphi_{1}=(0,1), \quad \varphi_{2}=\left(\frac{\sqrt{3}}{2},-\frac{1}{2}\right), \varphi_{3}=\left(-\frac{\sqrt{3}}{2},-\frac{1}{2}\right)$.

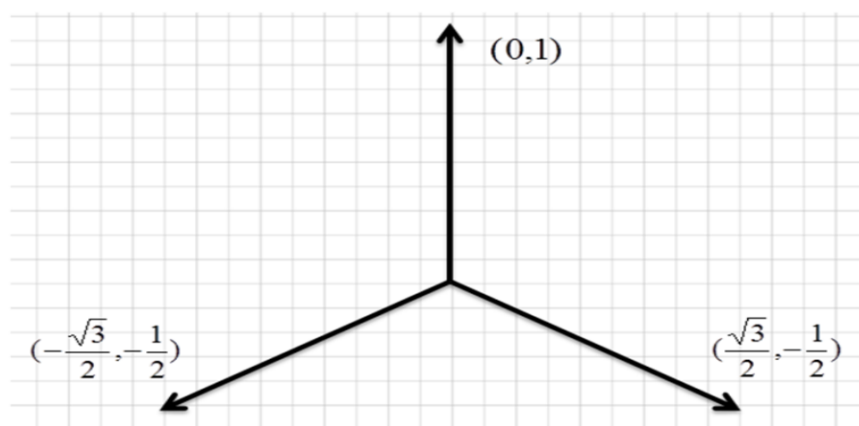

Fig. 2. The Mercedes-Benz Frame in $R^{2}$, consisting of 3 unit vectors located at an angle of $120^{\circ}$. 
Then fulfillment of the condition $\left|\left\langle x, \varphi_{i}\right\rangle\right|=\left\langle y, \varphi_{i}\right\rangle_{i=1}^{3}$ means that

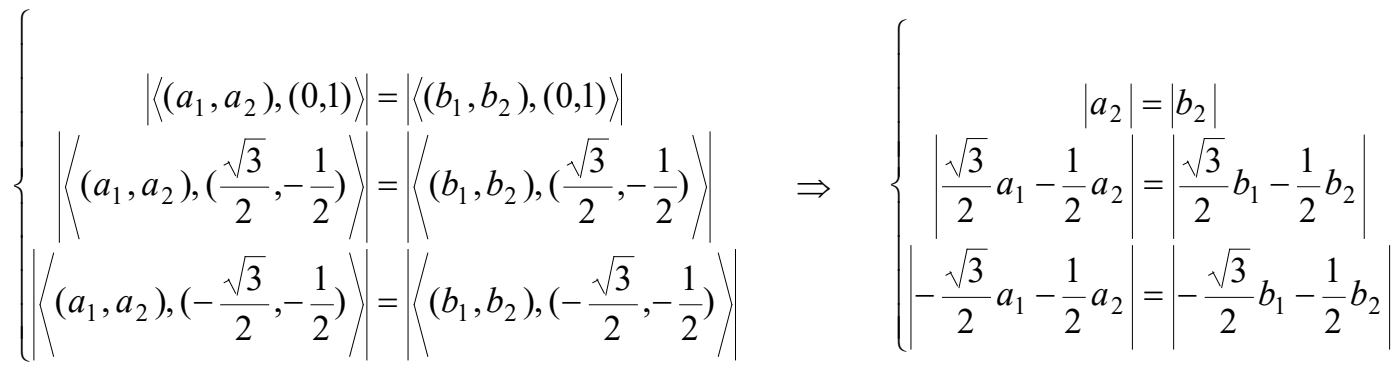

$$
\begin{aligned}
& \left\{\begin{array}{rl}
\left|a_{2}\right| & =\left|b_{2}\right| \\
\left|\sqrt{3} a_{1}-a_{2}\right| & =\left|\sqrt{3} b_{1}-b_{2}\right| \\
\left|\sqrt{3} a_{1}+a_{2}\right| & =\left|\sqrt{3} b_{1}+b_{2}\right|
\end{array} .\right.
\end{aligned}
$$

Squaring the equations of the last system we obtain that

$$
a_{1} a_{2}=b_{1} b_{2} \text { and } a_{1}^{2}=b_{1}^{2} \text {. }
$$

From this it follows that the first equality gives coincidence of the signs (to the multiplier), and the second - coincidence of the absolute values of coordinates. Moreover, from these equalities it also follows that zero coordinates are the same, if any.

We obtain that either $x=y$, or $x=-y$. Then there really exists $\theta= \pm 1$ such that if $\Phi=\left\{\varphi_{i}\right\}_{i=1}^{3}$ is the Mercedes-Benz frame, it does both phases reconstruction (because the vectors have the same signs, which means that the phases are the same too) and phaseless reconstruction (for $x=\theta y$ ) at the same time.

If we know the absolute values of coordinates of the vectors $x=\left(a_{1}, a_{2}\right)$ and $y=\left(b_{1}, b_{2}\right)$, then $x$ and $y$ can be one of 4 vectors (Fig. 3):

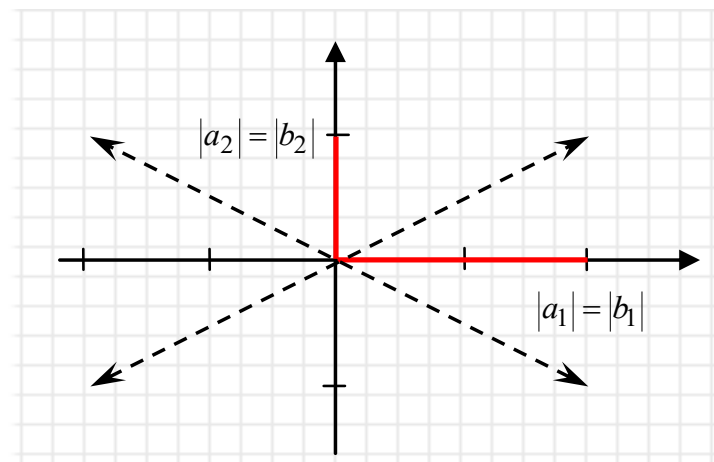

Fig. 3. Possible vectors for the known absolute values of coordinates of the vectors. $x, y$.

After scalar multiplication by the frame coordinates, the condition $a_{1} a_{2}=b_{1} b_{2}$ imposes restrictions on the signs of coordinates (Fig. 4):
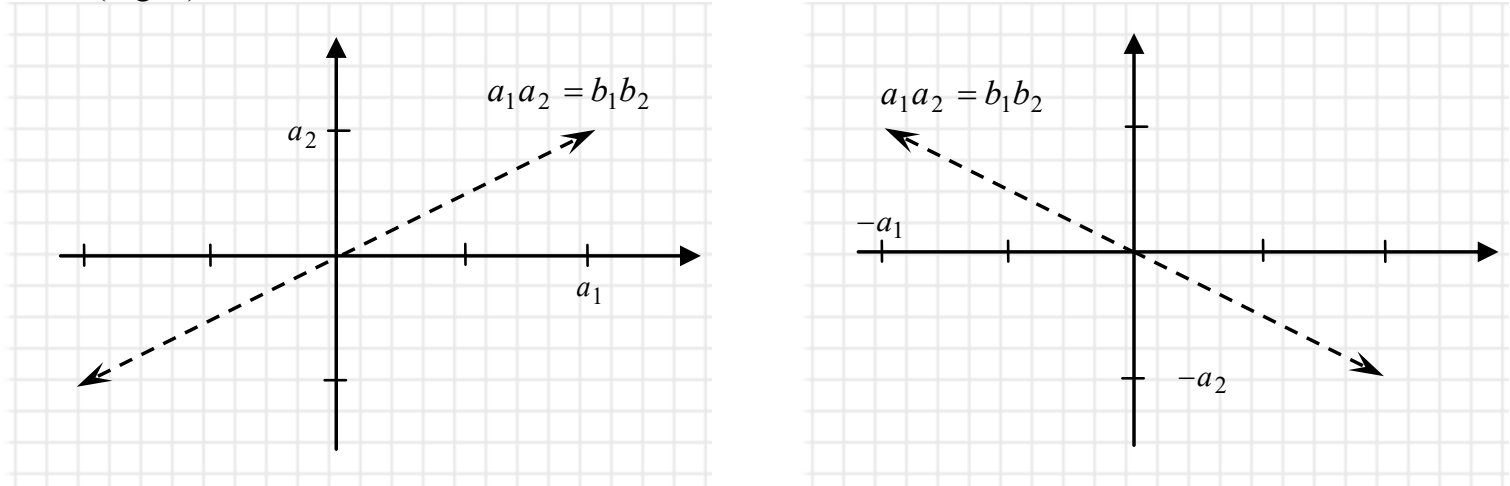

Fig. 4. Possible vectors for the known $\left|\left\langle x, \varphi_{i}\right\rangle,\right|\left\langle y, \varphi_{i}\right\rangle \mid$.

Let's consider an example in $C^{2}$. In the complex case:

$$
x=\left(x_{1}, x_{2}\right)=(a+i b, c+i d) \text { and } y=\left(y_{1}, y_{2}\right)=(e+i f, g+i h) \text {. }
$$

As $\Phi=\left\{\varphi_{i}\right\}_{i=1}^{5}$ let's take a frame of the following type:

$$
\varphi_{1}=(1,0), \varphi_{2}=\left(\frac{1}{\sqrt{2}},-\frac{1}{\sqrt{2}}\right), \varphi_{3}=\left(\frac{1}{\sqrt{2}}, \frac{1}{\sqrt{2}}\right), \varphi_{4}=\left(\frac{1}{\sqrt{2}}, \frac{1}{\sqrt{2}} i\right), \varphi_{5}=\left(\frac{1}{\sqrt{2}},-\frac{1}{\sqrt{2}} i\right) .
$$


Then fulfillment of the condition $\left|\left\langle x, \varphi_{i}\right\rangle\right|=\left.\left\langle y, \varphi_{i}\right\rangle\right|_{i=1} ^{5}$ means that

$$
\left\{\begin{array}{rl}
\mid\left\langle\left(x_{1}, x_{2}\right),(1,0)\right\rangle & =\left\langle\left(y_{1}, y_{2}\right),(1,0)\right\rangle \\
\left\langle\left(x_{1}, x_{2}\right),\left(\frac{1}{\sqrt{2}},-\frac{1}{\sqrt{2}}\right)\right\rangle & =\left\langle\left(y_{1}, y_{2}\right),\left(\frac{1}{\sqrt{2}},-\frac{1}{\sqrt{2}}\right)\right\rangle \\
\left\langle\left(x_{1}, x_{2}\right),\left(\frac{1}{\sqrt{2}}, \frac{1}{\sqrt{2}}\right)\right\rangle & =\left\langle\left(y_{1}, y_{2}\right),\left(\frac{1}{\sqrt{2}}, \frac{1}{\sqrt{2}}\right)\right\rangle \\
\left\langle\left(x_{1}, x_{2}\right),\left(\frac{1}{\sqrt{2}}, \frac{1}{\sqrt{2}} i\right)\right\rangle & =\left\langle\left(y_{1}, y_{2}\right),\left(\frac{1}{\sqrt{2}}, \frac{1}{\sqrt{2}} i\right)\right\rangle \\
\left\langle\left\langle\left(x_{1}, x_{2}\right),\left(\frac{1}{\sqrt{2}},-\frac{1}{\sqrt{2}} i\right)\right\rangle\right. & =\left\langle\left(y_{1}, y_{2}\right),\left(\frac{1}{\sqrt{2}},-\frac{1}{\sqrt{2}} i\right)\right\rangle
\end{array} .\right.
$$

Then $\left(x, \varphi_{i}\right)=\left\langle\left(x_{1}, x_{2}\right),\left(\varphi_{i 1}, \varphi_{i 2}\right)\right\rangle=\sum_{\substack{j=1,2 \\ i=1,5}} x_{j} \overline{\varphi_{i j}}$ and $\left|\left\langle x, \varphi_{i}\right\rangle\right|=\left.\left\langle y, \varphi_{i}\right\rangle\right|_{i=1} ^{5}$ mean that:

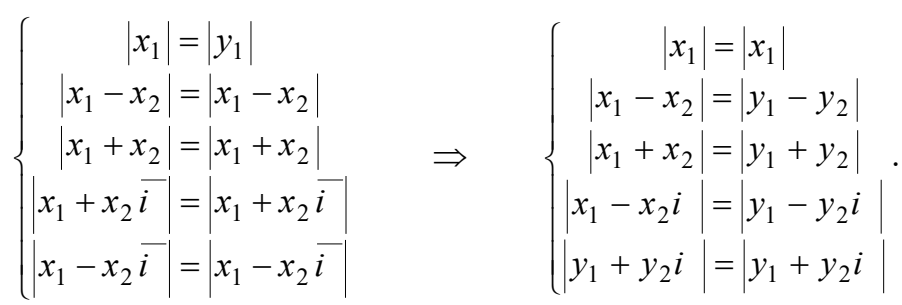

Let's rewrite the system in the following form:

$$
\left\{\begin{aligned}
a^{2}+b^{2} & =e^{2}+f^{2} \\
(a-c)^{2}+(b-d)^{2} & =(e-g)^{2}+(f-h)^{2} \\
(a+c)^{2}+(b+d)^{2} & =(e+g)^{2}+(f+h)^{2} \\
(a+d)^{2}+(b-c)^{2} & =(e+h)^{2}+(f-g)^{2} \\
(a-d)^{2}+(b+c)^{2} & =(e-h)^{2}+(f+g)^{2}
\end{aligned}\right.
$$

From the last system we obtain

$$
c^{2}+d^{2}=g^{2}+h^{2}
$$

And it means that the absolute values of the second complex coordinates of the vectors $x$ and $y$ are equal, because:

$$
c^{2}+d^{2}=\left|x_{2}\right|=g^{2}+h^{2}=\left|y_{2}\right| \text {. }
$$

So, if we take a frame of the type $\varphi_{1}=(1,0), \varphi_{2}=\left(\frac{1}{\sqrt{2}},-\frac{1}{\sqrt{2}}\right), \varphi_{3}=\left(\frac{1}{\sqrt{2}}, \frac{1}{\sqrt{2}}\right), \varphi_{4}=\left(\frac{1}{\sqrt{2}}, \frac{1}{\sqrt{2}} i\right), \varphi_{5}=\left(\frac{1}{\sqrt{2}},-\frac{1}{\sqrt{2}} i\right)$, then the absolute values of the corresponding complex coordinates of the vectors $x$ and $y$ are equal, i.e.:

$$
\left|x_{1}\right|=\left|y_{1}\right|=r_{1} \text { and }\left|x_{2}\right|=\left|y_{2}\right|=r_{2} \text {. }
$$

Now, let us write down the coordinates of the vectors in the polar form, taking into account the equality of the absolute values of the coordinates:

$$
\left.x=\left(x_{1}, x_{2}\right)=\left(r_{1} e^{i \cdot \varphi_{1}}, r_{2} e^{i \cdot \varphi_{2}}\right) \text { and } y=\left(y_{1}, y_{2}\right)=\left(r_{1} e^{i \cdot \psi_{1}}, r_{2} e^{i \cdot \psi_{2}}\right)\right) .
$$

Then $\left|\left\langle x, \varphi_{i}\right\rangle\right|=\left.\left\langle y, \varphi_{i}\right\rangle\right|_{i=1} ^{5}$ will look as follows:

$$
\left\{\begin{aligned}
\left|\left(r_{1} e^{i \cdot \varphi_{1}}, r_{2} e^{i \cdot \varphi_{2}}\right),(1,0)\right\rangle & =\left\langle\left(r_{1} e^{i \cdot \psi_{1}}, r_{2} e^{i \cdot \psi_{2}}\right),(1,0)\right\rangle \\
\left\langle\left(r_{1} e^{i \cdot \varphi_{1}}, r_{2} e^{i \cdot \varphi_{2}}\right),\left(\frac{1}{\sqrt{2}},-\frac{1}{\sqrt{2}}\right)\right\rangle & =\left\langle\left(r_{1} e^{i \cdot \psi_{1}}, r_{2} e^{i \cdot \psi_{2}}\right),\left(\frac{1}{\sqrt{2}},-\frac{1}{\sqrt{2}}\right)\right\rangle \\
\left\langle\left(r_{1} e^{i \cdot \varphi_{1}}, r_{2} e^{i \cdot \varphi_{2}}\right),\left(\frac{1}{\sqrt{2}}, \frac{1}{\sqrt{2}}\right)\right\rangle & =\left\langle\left(r_{1} e^{i \cdot \psi_{1}}, r_{2} e^{i \cdot \psi_{2}}\right),\left(\frac{1}{\sqrt{2}}, \frac{1}{\sqrt{2}}\right)\right\rangle \\
\left\langle\left(r_{1} e^{i \cdot \varphi_{1}}, r_{2} e^{i \cdot \varphi_{2}}\right),\left(\frac{1}{\sqrt{2}}, \frac{1}{\sqrt{2}} i\right)\right\rangle & =\left\langle\left(r_{1} e^{i \cdot \psi_{1}}, r_{2} e^{i \cdot \psi_{2}}\right),\left(\frac{1}{\sqrt{2}}, \frac{1}{\sqrt{2}} i\right)\right\rangle \\
\mid\left\langle\left(r_{1} e^{i \cdot \varphi_{1}}, r_{2} e^{i \cdot \varphi 2}\right),\left(\frac{1}{\sqrt{2}},-\frac{1}{\sqrt{2}} i\right)\right\rangle & =\left\langle\left(r_{1} e^{i \cdot \psi_{1}}, r_{2} e^{i \cdot \psi_{2}}\right),\left(\frac{1}{\sqrt{2}},-\frac{1}{\sqrt{2}} i\right)\right\rangle
\end{aligned}\right\rangle
$$

From this it follows that $\varphi_{1}=\psi_{1}$ and $\varphi_{2}=\psi_{2}$. We obtain that phases of the vectors $x$ and $y$ are equal to $2 \pi k, k \in \mathrm{Z}$. 


\section{Weak phase retrieval}

Definition 1. Two vectors $x=\left(a_{1}, a_{2}, \ldots, a_{m}\right)$ and $y=\left(b_{1}, b_{2}, \ldots, b_{m}\right)$ do weak phase retrieval if there is a $|\theta|=1$ such that phase $a_{i}=\theta$ phase $b_{i}$, for all $i=1,2, \ldots, m$, such that $a_{i} \neq 0 \neq b_{i}$.

In the real case, if $\theta=1$, we say that $x, y$ have weakly like signs and if $\theta=-1$ they have weakly opposite signs.

Definition 2. A family of vectors $\Phi=\left\{\varphi_{i}\right\}_{i=1}^{n}$ in $H^{\mathrm{m}}$ does weak phase retrieval if from equalities

$$
\left|\left\langle x, \varphi_{i}\right\rangle=\left\langle y, \varphi_{i}\right\rangle\right|, i=1,2, \ldots, n \text {. }
$$

It follows that there exists a $|\theta|=1$, such that

$$
\text { phase } x_{i}=\theta \text { phase } y_{i} \text {, for all } i=1,2, \ldots, m \text {, so that } a_{i} \neq 0 \neq b_{i} \text {. }
$$

The weak phase retrieval differs from the phase retrieval described in Definition 2 of Section 4 in that there can be both $a_{i}=0$ and $b_{i} \neq 0$.

Let us consider an example where the weak phase retrieval is done but the phase retrieval by Definition 2 of Section 4 is failed. Let us consider in $R^{\mathrm{m}}$ a set of vectors $\Phi=\left\{\varphi_{i}\right\}_{i=1}^{m+1}$, which coordinates form the following matrix columns:

$$
A=\left(\begin{array}{ccccc}
1 & -1 & 1 & 1 & 1 \\
1 & 1 & -1 & 1 & 1 \\
1 & 1 & 1 & 1 & 1
\end{array}\right)_{m \times(m+1)} .
$$

Then for every $x=\left(a_{1}, a_{2}, \ldots, a_{m}\right)$ and $y=\left(b_{1}, b_{2}, \ldots, b_{m}\right)$, if $\left|\left\langle x, \varphi_{i}\right\rangle\right|^{2}=\left|\left\langle y, \varphi_{i}\right\rangle\right|^{2}$, it follows that $a_{i} a_{j}=b_{i} b_{j}$ for all $i \neq j$.

This set of $(m+1)$-vectors in $R^{\mathrm{m}}$ will do weak phase retrieval.

Theorem 1: Let $x=\left(a_{1}, a_{2}, \ldots, a_{m}\right)$ and $y=\left(b_{1}, b_{2}, \ldots, b_{m}\right)$ be two vectors in $R^{\mathrm{m}}$. Then the following statements are equivalent:

1) $\operatorname{sgn}\left(a_{i} a_{j}\right)=\operatorname{sgn}\left(b_{i} b_{j}\right)$, for all $1 \leq i \neq j \leq m$.

2) $x, y$ have either weakly like signs or weakly opposite signs.

\section{Conclusion}

In case the phase information is not available, the signal recovery is theoretically possible if redundant systems called frames are used as the system of representation. A well-chosen frame can provide numerical stability for recovery of the signal and obtaining important characteristics of the signal.

In the real case under certain conditions a generic frame consisting of (2m-1)-vectors can do phaseless reconstruction. In the complex space a generic frame consisting of (4m-2)-vectors can do the same under certain conditions. A family of frames recovers the signal by the absolute value of frame coefficients in polynomial time. The issue of the equivalence of phases retrieval and phaseless reconstruction is raised and partially resolved. Examples of signal recovery in small-dimension spaces are given.

A search and theoretical justification of new methods of recovery of information hidden in phases of transmitted signals and unavailable for measurements by publicly available physical instruments is conducted. The technique is based on the latest achievements in the research of complete linearly dependent systems called space frames.

\section{Acknowledgements}

The authors thank S.Ya. Novikov for his fruitful discussions.

\section{References}

[1] Botelho-Andrade S, Casazza P, Van Nguyen H, Tremain J. Phase retrieval verses phaseless reconstruction. ArXiv:1507.05815 [math.FA] - 21 Jul 2015.

[2] Shchelokov EA. Application of technologies of wireless data transmission on aerospace hardware. The Bulletin of the Ryazan state radio engineering university 2016; 56: 131-135

[3] Bandeira A, Cahill J, Mixon D, Nelson A. Saving phase: Injectivity and stability for phase retrieval. Applied and Computational Harmonic Analysis (ACHA) 2014; 37(I.1): 106-125.

[4] Balan R, Bodmann BG, Casazza PG, Edidin D. Fast algorithems for signal reconstruction without phase. Proceedings of SPIE-Wavelets XII, San Diego 2007; 6701: 670111920-670111932.

[5] Balan R, Casazza P, Edidin D. On signal reconstruction without phase. Appl. Comput. Harmon. Anal. 2006; 20: $345-356$.

[6] Holmes R, Paulsen VI. Optimal frames for erasures. Lin. Alg. Appl. 2004; 377: 31-51.

[7] Balan R, Bodman BG, Casazza PG, Edidin D. Painless reconstruction from magnitudes of frame coefficients, preprint.

[8] Novikov SYa, Fedina ME. Complete systems in problems of signal reconstruction. Proceedings of the International Scientific and Technical Conference. Vol. 1 . Perspective Information Technologies 2015; 280-283. (in Russian)

[9] Cameron PJ, Seidel JJ. Quadratic forms over GF(2). Indag. Math. 1973; 35: 1-8. 
Mathematical Modeling / A.A. Kuleshova, E.A. Shchelokov

[10] Cahill J, Mixon DG. Full Spark Frames. Available online: arXiv:1110.3548.

[11] Kuleshova A. Generic frame in problems for signal reconstruction without phase. Information Technology and Nanotechnology 2016, Ceur WS 2016.; $1638 ; 364-372$. 